\title{
Interpretation of the causes of instability of flash visual evoked potentials in intraoperative monitoring and proposal of a recording method for reliable functional monitoring of visual evoked potentials using a light-emitting device
}

\author{
Atsushi Sato, MD \\ Division of Neurosurgery, Ina Central Hospital, Ina, Japan
}

\begin{abstract}
OBJECTIVE Effective monitoring and application of visual evoked potentials (VEPs) during neurosurgery is a major challenge. While many monitoring methods have been effectively used, the use of VEPs as an objective determination method has not been established. The purpose of this report was to present a method for overcoming this limitation according to the use of a specific stimulus.
\end{abstract}

METHODS Data analysis was performed in 26 cases of brain surgery. Observation was carried out for 2 groups of responses: the response derived from the start of light emission, described as the on response, and the response derived from the end of light emission, described as the off response. These reactions were separated by extending the light emission time. The waves from the visual cortex were selected from each reaction following the start and the end of light emission with consideration for the characteristics of the potential distribution. The waves were observed to characterize changes resulting from variations in duration and quantity of light emission. The results of the analysis were used to determine the optimal emission time and amount of light for effective use of wave components during VEP monitoring.

RESULTS Stable and recordable waves were observed by monitoring the off response, consisting of the P1-N1-P2 component, with a wave latency of approximately $100 \mathrm{msec}$. Since the off response was correlated with the input, the stable wave derived from the off response could be adjusted by changing the light emission time and intensity. Individual differences in the latency of the off response were decreased by extending the light emission time and reducing the quantity of light. However, it was difficult to achieve stability by adjusting the light intensity and emission time using the on response. The off response was confirmed to be sufficiently stable for intraoperative monitoring. Moreover, during 1 case in which manipulation of the optic nerve was necessary, reduction in the off response was found to occur when the nerve was manipulated and to reverse when the manipulation stopped.

CONCLUSIONS The off response was shown to have the capacity to function as a monitoring tool, providing more stable wave forms than the on response. Recording conditions could be adjusted to achieve a light-emitting time of 500 msec and a light quantity of $8000 \mathrm{Lx}$. Stable monitoring of VEPs using light-emitting stimuli can contribute toward improving surgical outcomes.

http://thejns.org/doi/abs/10.3171/2015.10.JNS151228

KEY WORDS intraoperative monitoring; visual evoked potential; neurosurgery; off response; surgical technique

$\mathrm{D}$ AMAGE to visual function is a major complication of brain surgery. While it is possible to monitor various functions intraoperatively, no effective method for visual monitoring has been developed to date. The reason for these difficulties, however, is not clear. The earliest studies of visual evoked potentials (VEPs), performed in
1964, showed that waves could be evoked using a light stimulus. Several stimulation methods for VEPs have been described,,$^{13,23}$ but only the most rudimentary stimuli (e.g., emission stimuli) can be applied during VEP recording. Thus, functional monitoring to evaluate the use of this limited stimulation method is challenging. Although

ABBREVIATIONS VEP $=$ visual evoked potential.

SUBMITTED May 27, 2015. ACCEPTED October 7, 2015.

INCLUDE WHEN CITING Published online February 19, 2016; DOI: 10.3171/2015.10.JNS151228. 
the possibility of achieving intraoperative monitoring of VEPs has been investigated extensively, ${ }^{25}$ attempts have been unsuccessful in many studies. ${ }^{1-5,24}$ The reasons for these inconsistencies include difficulties reproducing results, unexpected wave changes, and individual differences. ${ }^{17,19}$ Indeed, no studies have reported typical wave patterns that can be repeatedly observed with VEP monitoring. $6,7,9,12,14,15,18$ In contrast, some have suggested that the effectiveness of VEP monitoring may be improved by altering the stimulation device and light quantity. $8,10,11,21,22$ In order for VEPs to be used as a monitoring tool, a specific wave must be defined, preferably with little or no individual difference and sufficiently high reproducibility. Furthermore, the amplitude and peak latency of the wave must be related to the size of the stimulation input. To date, no methods for recording or monitoring of intraoperative VEPs have met these requirements. However, no studies have reported the effects of variations in light stimulation on wave stability and reproducibility.

During anesthesia, accessible optical stimulation to the retina is quite limited. While conventional studies have only used flash stimulation as the input for VEP monitoring, researchers have hypothesized that changes in the stimulation may allow this problem to be effectively addressed because reliable factors may be difficult to discern or varied in the wave of flash VEPs. The factors that can be varied are also limited. The emission stimulation is established by 2 components - intensity and time of light emission. Thus, adjustment of these 2 elements may allow for removal of unstable elements and extraction of reliable waves. The resulting data would allow for division based on light stimulation into an on state (during which light is emitted) and an off state (during which no light is emitted), with separations of the specific characteristics of the waves.

In this study, analysis of 2 reaction waves allowed for analysis of the causes of VEP instability using conventional stimulation with a flash of light. Additionally, a stimulation method in which VEPs could be used as a monitoring tool is also described.

\section{Methods}

Multiple steps were required to effectively identify the waves in the target (Fig. 1). The light stimulation involved a combination of on and off characteristics. Continuous flashes allowed for separation of the response patterns, and each response was recordable as a light emission response (termed the on response) or a no-emission response (termed the off response). Both the on response and the off response needed to be considered based on the change in the duration of light emission.

The verification process was as follows. First, sufficient separation of the on response and off response was achieved by lengthening the light emission time. Next, a typical waveform was established for each of the respons-

\section{Stimulation Setting 1}

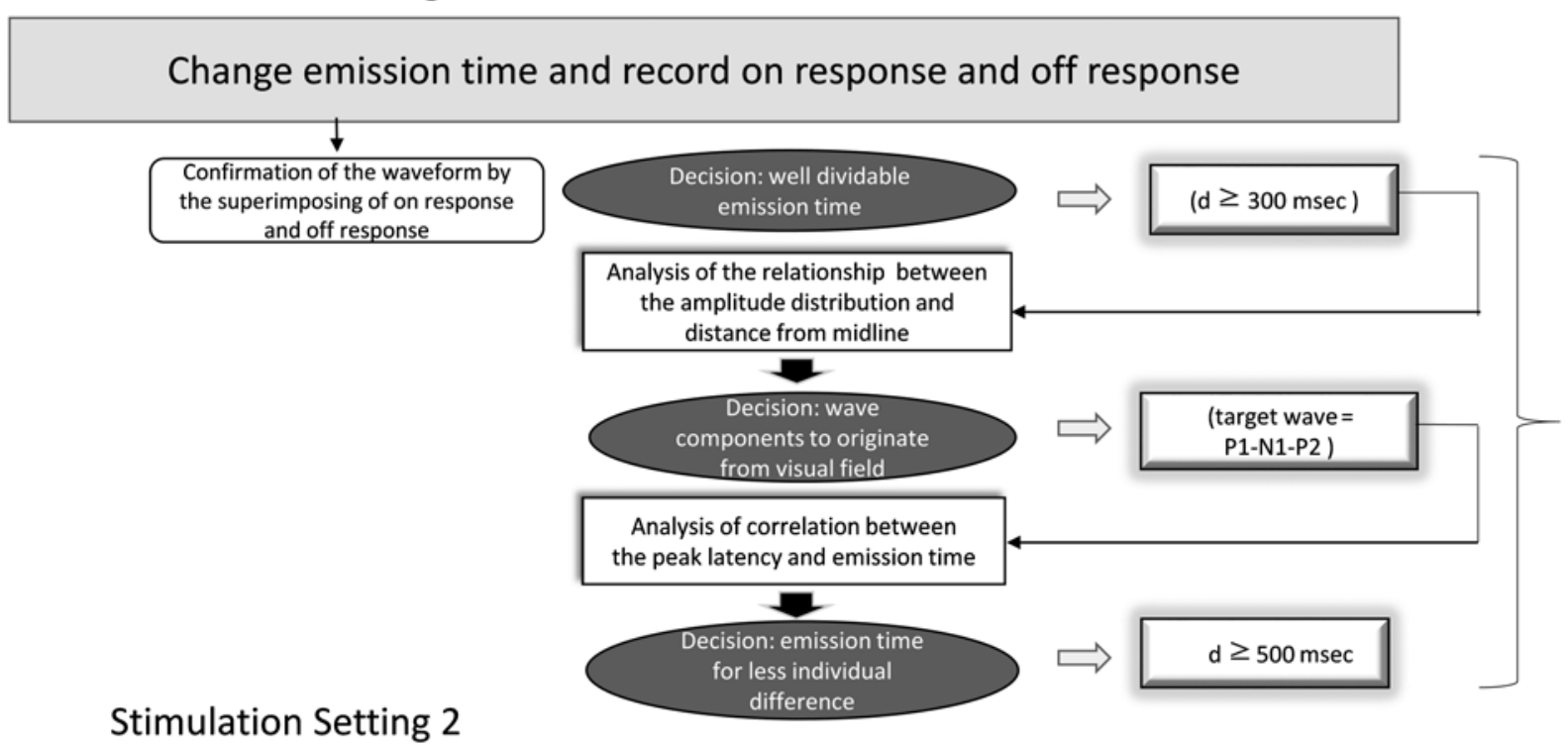

Change emission quantity and record on response and off response

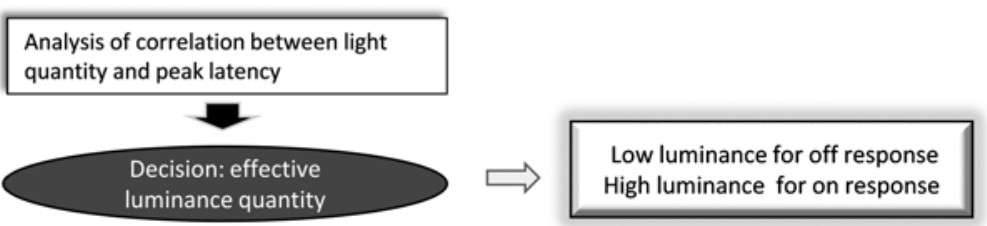

FIG. 1. Schematic showing the validation process for determining the wave to be monitored. 
es. The wave from the primary visual cortex was then estimated using the components of each waveform. During the peak latency of the waves, the effects of changes in the light emission time were monitored. Finally, the effects of the latency were analyzed by changing the amount of light emitted at a fixed emission time. The reproducible components of the wave were extracted, and their latency and amplitude were measured.

For initial examination, intraoperative VEPs were recorded in 26 patients (10 men and 16 women, mean age 59.9 years, range 19-90 years) who underwent brain surgery under general anesthesia. All surgical procedures were performed under propofol anesthesia. None of the patients had any preoperative visual disturbance. The preoperative diagnoses were as follows: 17 patients had unruptured aneurysms, 4 patients had brain tumors (1 left frontal glioma, 2 convexity meningiomas, and 1 hemangiopericytoma in the left cerebellar hemisphere), 2 patients had arterial stenosis, 2 patients had Chiari malformation, and 1 patient had trigeminal neuralgia. All patients had conditions that were not expected to involve visual changes during surgery. All patients provided written, informed consent for participation in the study. The study protocol was approved by the institutional review board of Ina Central Hospital.

A silicon stimulation device with an embedded diode for the light stimulus (Unique Medical Co.) was patched on the eyelid with the eye closed, and the patients' eyes were then covered with a light-shielding sheet (Fig. 2A). The maximum potential light emission of the stimulation device was 40,000 Lx. Therefore, the maximum amount of light was set as 20,000 Lx. A Bath Amplifier (Nihon Kohden Co.) was used to control the quantity of photic stimulation. The emission time was controlled by an electronic stimulator (Nihon Kohden Co.). Each evoked potential was the average of 100 responses, and each stimulation was recorded twice. The stimulus frequency was $1 \mathrm{~Hz}$. The evoked potentials were triggered by switching on the light and were amplified using Neuropack (Nihon Kohden Co.). The analysis time was $1000 \mathrm{msec}$, and the bandwidth was set at 0.1-200 Hz. Evoked potentials were recorded from the electrodes at a total of 7 poles (R3, R2, R1, MO, $\mathrm{L} 1, \mathrm{~L} 2$, and L3). The midline electrode was placed $1 \mathrm{~cm}$ above the inion, and the lateral electrodes were placed at the same level at intervals of $1.5 \mathrm{~cm}$, as shown in Fig. 2 . Lined bilateral earlobe electrodes were used for reference.

First, the emission time was changed with a fixed light quantity of 20,000 Lx. Both eyes were stimulated, and each reaction waveform was recorded. Then, with a fixed light emission time, the amount of light was successively decreased from the maximum of $20,000 \mathrm{Lx}$ to $15,000 \mathrm{Lx}$ and finally to $10,000 \mathrm{Lx}$ and the reactions to these changes were recorded (Fig. 1).

VEP recording can be highly influenced by the method of anesthesia. ${ }^{16,20,24}$ Therefore, all recordings were performed under propofol anesthesia. ${ }^{16}$ The data show the responses recorded when the stimulus (light) reached the retina through the eyelid in the closed-eye state, similar to light stimulus by application of a red filter. The pupil diameter and eye position were not controlled, but the method of anesthesia and recording site stimulation methods
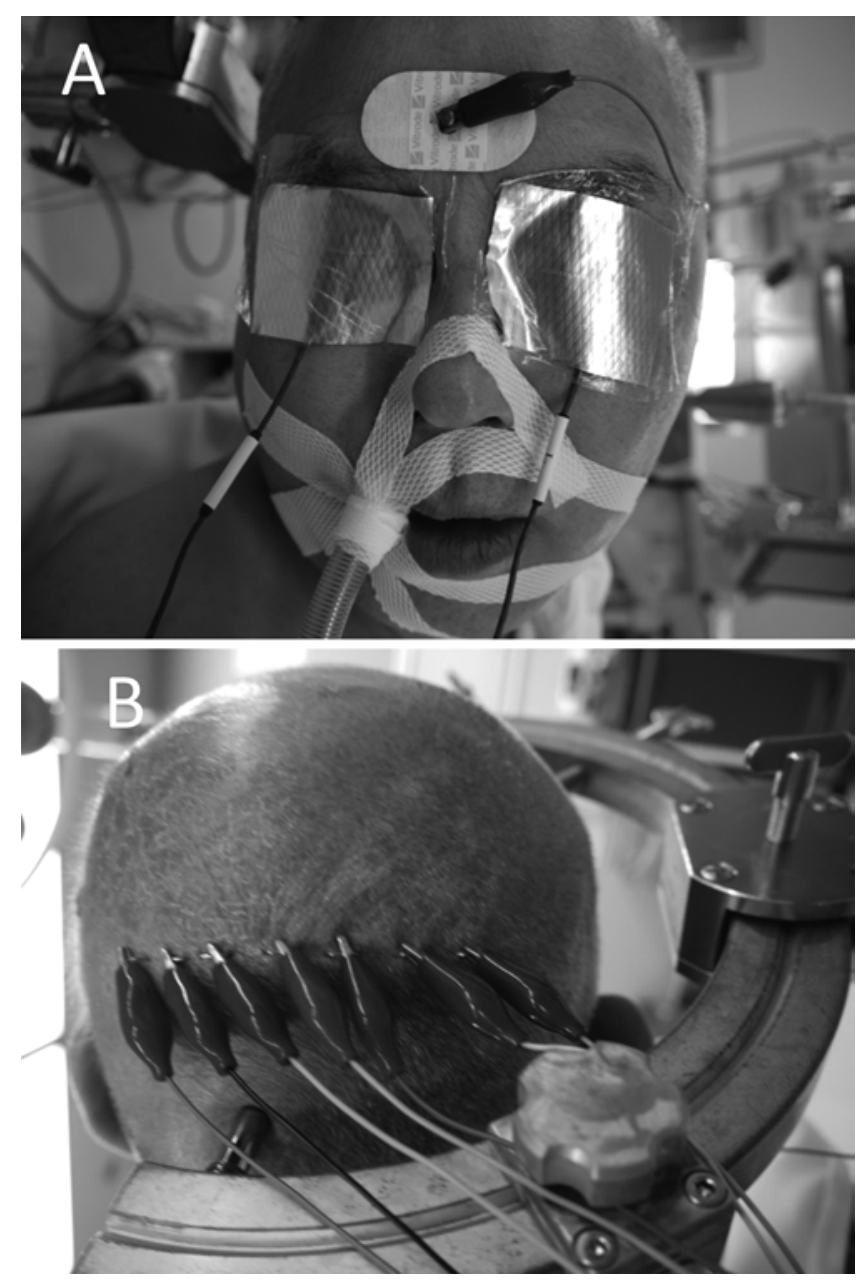

FIG. 2. A: Image showing the device settings. B: Image showing the location of 7 electrodes on the skin overlying the occiput.

were consistent between individuals. Recording based on changes in the emission time or light quantity was completed before the beginning of intracranial manipulation. Light quantity was determined based on the light-emitting stimulator and was not necessarily the same as the quantity of light reaching the retina. Thus, the conditions used herein were the same as those used during general neurosurgery, and no special configuration was required for recording.

\section{Analysis}

Waves that were common to each patient were numbered such that the upward wave was negative and the downward wave was positive. The peak-to-peak amplitude was measured for each wave component. The basic model for waveform evaluation assumes that there is a primary visual cortex in the back of the head at the midline of the spherical cranium. The wave components from the generator in the visual cortex located in the occipital midline will have maximum amplitude in the midline electrode, with the amplitude decreasing along the outer parts of the recording point. The target wave derived from the visual cortex was selected based on this principle. The latency of the vertices of the waves that were extracted 
from the recording of the midline electrode was analyzed to determine how the wave changed with changes in emission time or quantity.

\section{Statistical Analysis}

The relationship between the distance from the midline electrode and amplitude of the wave components recorded to each electrode was determined using regression analysis. Then, the amplitude of the wave component was reduced such that when the distance from the midline was greater, a clear negative coefficient could be confirmed. The amplitude of the peak-to-peak area was defined as the objective value, and the light emission time and measuring point (the distance from the midline) were defined as the explanation variables to execute multivariable analysis. At this time, while considering individual differences in amplitude, normalization of the measured amplitude was performed. The amplitudes for 7 points were normalized to the unit norm according to the formula below:

$$
\frac{x_{1}}{d}, \frac{x_{2}}{d}, \ldots, \frac{x_{7}}{d}, d=\sqrt{\sum_{i=1}^{7} x_{i}^{2}}
$$

where $x$ is the measured amplitude.
The normalized data were entered into a statistical program ( $\mathrm{R}$ version 3.1.0, $\mathrm{R}$ Core Team) to calculate the multiple correlation coefficient. This calculation enabled the determination of the component of the waveform of the midline origin if there was a negative correlation with respect to the distance from the midline in the regression model. The statistical difference between emission time and peak latency was analyzed using Mann-Whitney tests. The significance level was set to less than 0.0083 , based on the Bonferroni correction. Relationships between light emission quantity and latency were confirmed by Wilcoxon signed-rank test. The significance level was set to less than 0.025 , based on the Bonferroni correction.

\section{Results}

Overlay waves recorded from the midline electrode in each case were used to clarify the features of the on and off responses. Separation of the on response and off response could be achieved when the light emission time was more than $300 \mathrm{msec}$ (Fig. 3). The common features of the waveforms for each patient were determined. The on response was characterized by waveforms with 4 peaks around 100 msec, while the off response was characterized by a simple wave with 3 peaks observed at around 100 msec. Typical waveforms are shown in the schematic diagram in Fig. 4.
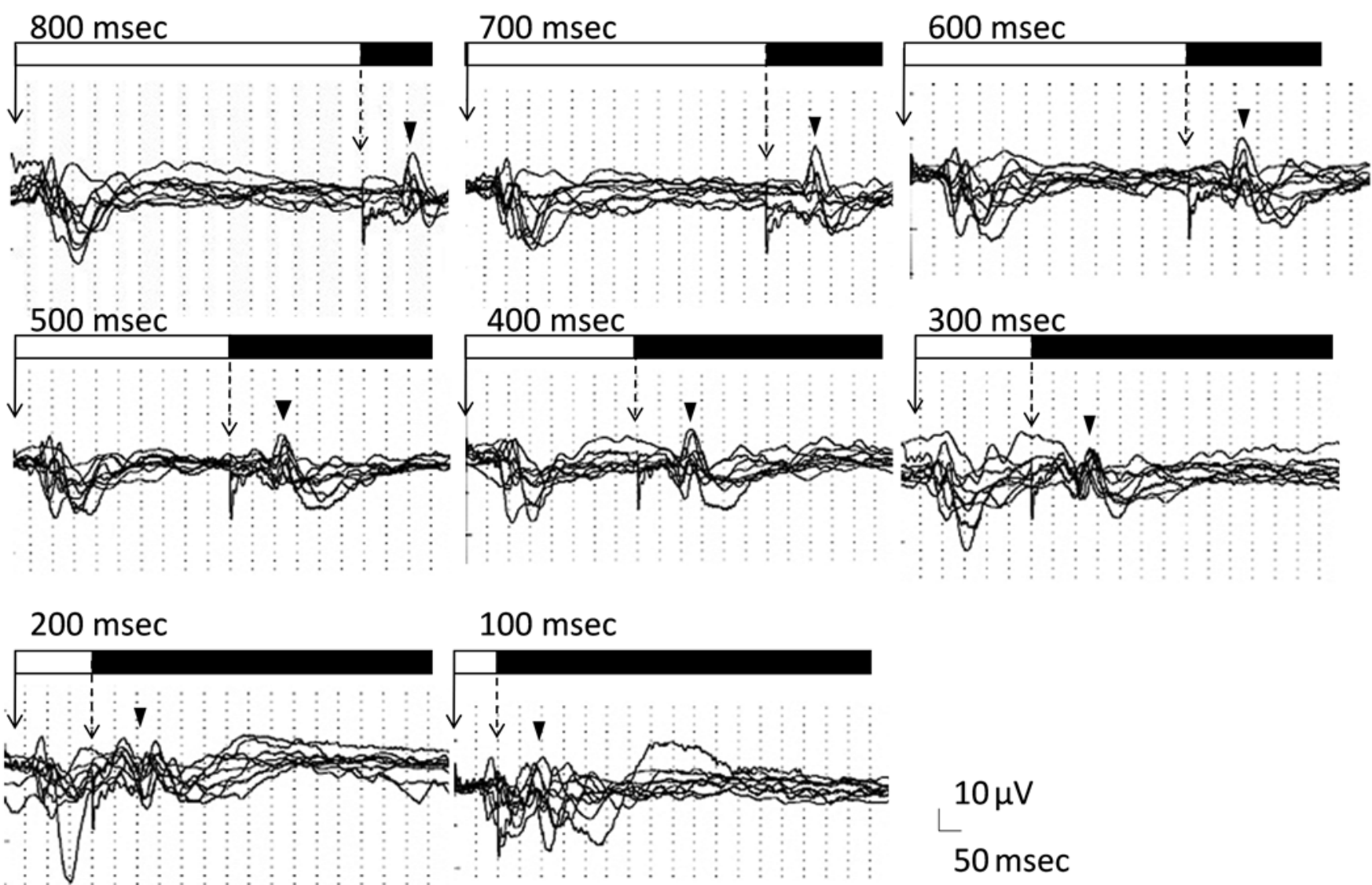

FIG. 3. Overlay waveforms of recordings at the $\mathrm{MO}$ electrode in 10 randomly chosen cases. A moderate stimulating pattern is shown by the upper bar. The white area of the bar shows the light-emission time, and the black area of the bar shows the time without light emission. For each image, the arrow on the left indicates the start of light emission, and the dashed arrow indicates the end of emission. The black arrowheads indicate the "off response." 


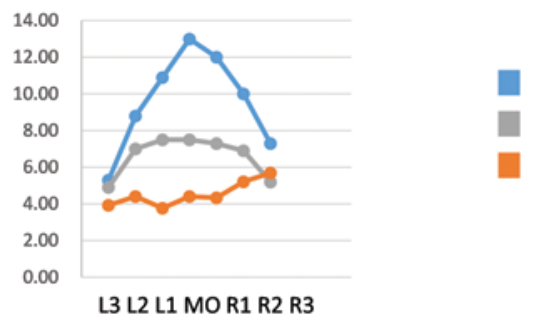

\section{Distribution of midline origin Incomplete distribution of midline origin Asymmetric distribution}
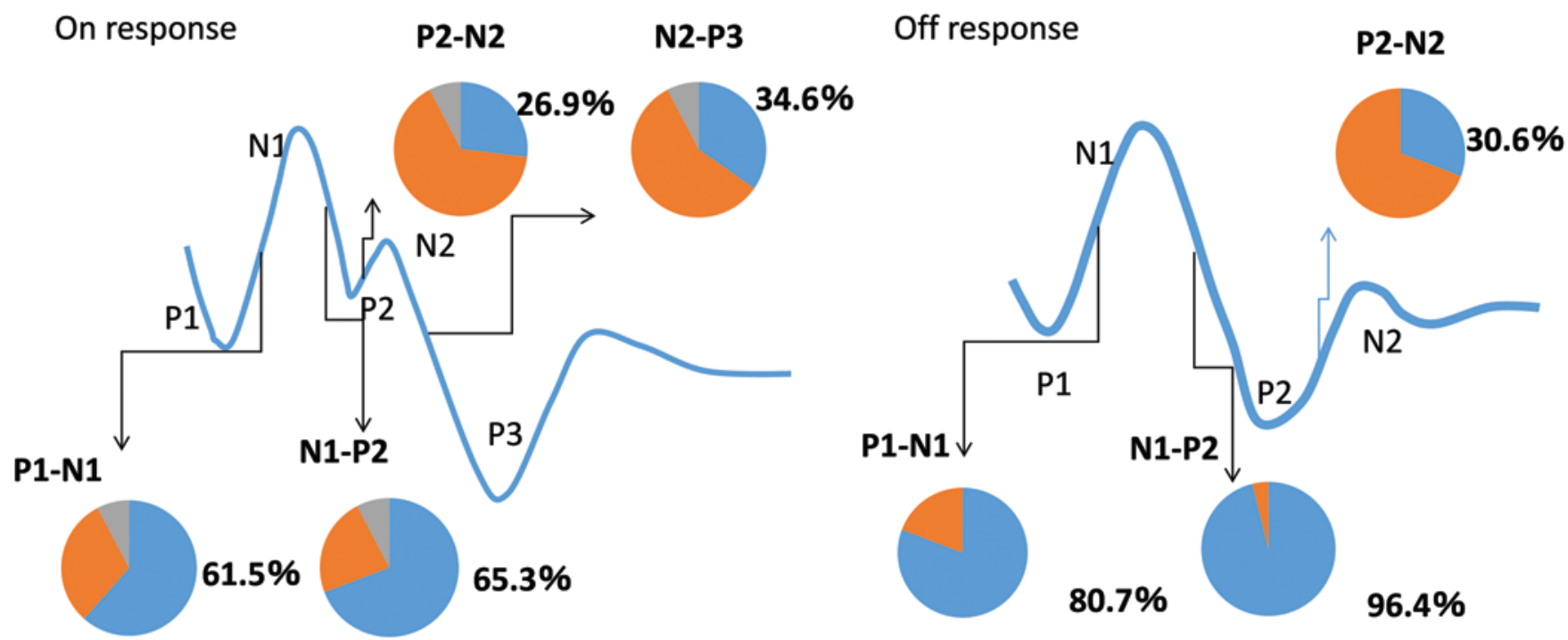

FIG. 4. The upper panel shows an image of the amplitude of each electrode. The lower panel shows typical waveforms for the on response and off response. The pie charts show the percentage of mound-shaped distribution (blue), incomplete mound-shaped distribution (gray), and asymmetrical distribution (orange) for each component.

The peaks of each waveform were named N1, P1, N2, $\mathrm{P} 2, \mathrm{~N} 3$, and P3, and the latency of each peak was measured. In addition, the peak-to-peak amplitudes of N1-P1, $\mathrm{P} 1-\mathrm{N} 2$, and N2-P2 (plus that of P2-N3 for the on response) were measured.

Multivariable analysis was carried out by normalization of the amplitude as the objective value and by using the measuring point and light emission time as the explanation values (Table 1). Because the coefficient for each amplitude was negative and the $p$ values obtained from Wald tests were sufficiently small, the data showed that the amplitude tended to increase as the distance from the center decreased. The measuring point contributed significantly as an explanation variable for the objective variable, while the light-emitting time did not. The contribution ratio $\left(\mathrm{R}^{2}\right)$ was highest in the model for N1-P2 for each off and on response. The N1-N2 amplitude was interpreted as susceptible to difference in the measured region. The sitespecific potential of the amplitude between the vertices was plotted as a component of the wave on the graph (see upper panel in Fig. 4). The percentage of waves for which the distribution of amplitude decreased from the highest center toward both ends with a $500-\mathrm{msec}$ emission time is shown in Fig. 4.

The shape distribution of the amplitude had to meet 3 conditions: the highest potential had to be achieved for the center electrode, the potential had to decrease as it went outward, and the potential difference between the sym- metrical electrodes had to be less than $2 \mu \mathrm{V}$. When the potential difference between the center electrode and the outermost electrode was less than $2 \mu \mathrm{V}$, the distribution was assumed to be incomplete (Fig. 4). This shape distribution was most frequently observed in N1-P2 of the off response (96.4\%) and was also commonly observed in $\mathrm{P} 1-\mathrm{N} 1$ of the off response (80.7\%). In the on response, this distribution was also confirmed in P1-N1 (61.5\%) and N1P2 (65.3\%; Fig. 4). The percentage of the mound-shaped distribution on the subsequent wave was dramatically reduced.

Because there were no differences in the time of peak latency at each electrode, the effects of the amount of light and the light-emitting time on the time of peak latency were measured by the midline electrode. Additionally, the effects of differences in light emission time on peak latency were confirmed in the off response (see upper panel of Fig. 5). Analysis of the differences in light emission times revealed that variations in individual differences decreased as the light-emitting time was increased and was associated with longer latency in peaks P1 and N1. However, in the on response, the emission duration did not significantly affect peak latency. Shortening of peak latency in the off response and reduced changes in latency were observed as the emission time increased (see upper panel in Fig. 5).

Results of different intensities with a fixed emission time of 500 msec showed varying effects on latency for 
TABLE 1. Multivariate analysis results for the amplitude*

\begin{tabular}{|c|c|c|c|c|c|c|}
\hline \multirow[b]{2}{*}{ Wave Component \& Variable } & \multicolumn{3}{|c|}{ Coefficient } & \multicolumn{2}{|c|}{ ANOVA } & \multirow[b]{2}{*}{$\mathrm{R}^{2}$} \\
\hline & Estimate & $95 \% \mathrm{Cl}$ & Wald Test & Contribution & F Test & \\
\hline Off P1-N1 & & & & & & 0.336 \\
\hline Intercept & 0.476 & $0.459-0.494$ & $3.029 \mathrm{E}-312$ & & & \\
\hline Points & -0.068 & -0.073 to -0.063 & $9.474 \mathrm{E}-108$ & $33.6 \%$ & $9.474 \mathrm{E}-108$ & \\
\hline Lighting time & 0.000 & $0.000-0.000$ & $8.051 \mathrm{E}-01$ & $0.0 \%$ & $8.051 \mathrm{E}-01$ & \\
\hline Off N1-P2 & & & & & & 0.479 \\
\hline Intercept & 0.504 & $0.487-0.521$ & $0.000 \mathrm{E}+00$ & & & \\
\hline Points & -0.087 & -0.092 to -0.082 & $2.589 \mathrm{E}-170$ & $47.9 \%$ & $2.589 \mathrm{E}-170$ & \\
\hline Lighting time & 0.000 & $0.000-0.000$ & $9.654 \mathrm{E}-01$ & $0.0 \%$ & $9.654 \mathrm{E}-01$ & \\
\hline Off P2-N2 & & & & & & 0.085 \\
\hline Intercept & 0.395 & $0.362-0.427$ & $1.304 \mathrm{E}-91$ & & & \\
\hline Points & -0.036 & -0.046 to -0.027 & $7.420 \mathrm{E}-14$ & $8.1 \%$ & $7.420 \mathrm{E}-14$ & \\
\hline Lighting time & 0.000 & $0.000-0.000$ & $1.015 \mathrm{E}-01$ & $0.4 \%$ & $1.015 \mathrm{E}-01$ & \\
\hline On P1-N1 & & & & & & 0.199 \\
\hline Intercept & 0.442 & $0.422-0.461$ & $2.082 E-255$ & & & \\
\hline Points & -0.052 & -0.058 to -0.047 & $3.835 \mathrm{E}-60$ & $19.9 \%$ & $3.835 E-60$ & \\
\hline Lighting time & 0.000 & $0.000-0.000$ & $4.365 \mathrm{E}-01$ & $0.0 \%$ & $4.365 \mathrm{E}-01$ & \\
\hline On N1-P2 & & & & & & 0.238 \\
\hline Intercept & 0.468 & $0.448-0.487$ & 1.897E-265 & & & \\
\hline Points & -0.057 & -0.063 to -0.051 & $1.819 \mathrm{E}-68$ & $23.7 \%$ & 1.819E-68 & \\
\hline Lighting time & 0.000 & $0.000-0.000$ & $1.478 \mathrm{E}-01$ & $0.1 \%$ & $1.478 \mathrm{E}-01$ & \\
\hline On P2-N2 & & & & & & 0.016 \\
\hline Intercept & 0.384 & $0.348-0.419$ & $1.631 \mathrm{E}-85$ & & & \\
\hline Points & -0.022 & -0.033 to -0.012 & $5.021 \mathrm{E}-05$ & $1.5 \%$ & $5.021 \mathrm{E}-05$ & \\
\hline Lighting time & 0.000 & $0.000-0.000$ & $1.974 \mathrm{E}-01$ & $0.1 \%$ & $1.974 \mathrm{E}-01$ & \\
\hline On N2-P3 & & & & & & 0.044 \\
\hline Intercept & 0.399 & $0.380-0.418$ & $1.979 \mathrm{E}-231$ & & & \\
\hline Points & -0.021 & -0.027 to -0.015 & $1.438 \mathrm{E}-12$ & $4.4 \%$ & $1.438 \mathrm{E}-12$ & \\
\hline Lighting time & 0.000 & $0.000-0.000$ & $9.540 \mathrm{E}-01$ & $0.0 \%$ & $9.540 \mathrm{E}-01$ & \\
\hline
\end{tabular}

* The objective variable is amplitude. The explanation variables are the measuring point (points) and the light emission time (lighting time).

the on and off responses. The intensities were 20,000 (100\%), 15,000 (75\%), and 10,000 Lx (50\%). The latency and individual differences in peak latency were reduced with lower quantities of light for the off response at P1 and N1. Conversely, for the on response, there was a tendency of the latency stability in N1 and P2 to increase as the amount of light increased (see lower panel in Fig. 5).

In the following cases, the actual wave change of the off response was observed intraoperatively.

\section{Illustrative Cases}

\section{Case 1}

This patient was a 61-year-old woman undergoing clip ligation of an unruptured left parasellar aneurysm via a left frontotemporal approach. In the surgical field, the aneurysm was hidden by the left optic nerve, and the surgeon had to elevate the optic nerve temporarily to confirm the aneurysm. VEP monitoring was stable with an emission time of $400 \mathrm{msec}$, emission quantity of $10000 \mathrm{Lx}$, and left single-eye stimulation. The waveform of the off response was changed by optic nerve manipulation. When the optic nerve was elevated gently (Fig. 6B), the amplitude decreased, and the latency of the peaks was delayed. For the MO electrode, the amplitude of P1-N1 in the off response decreased from 6.5 to $3.1 \mu \mathrm{V}$, and that of N1-P2 decreased from 7.3 to $5.4 \mu \mathrm{V}$. The peak latencies for P1, N1, and P2 were also delayed from 91 to $97.5 \mathrm{msec}$, from 97.5 to 110 $\mathrm{msec}$, and from 129 to $143 \mathrm{msec}$, respectively. This phenomenon was reversed by stopping the manipulation (Fig. 6C). Care was taken to avoid optic nerve manipulation for the rest of the surgical procedure, and the wave remained stable. At the end of the operation, after closure of the incision, VEPs were recorded using the artificial limitation of the input. The amplitude of the off response decreased logarithmically with the decrease in emission quantity, and peak latency was prolonged logarithmically with the decrease in emission quantity (Fig. 6 right). The patient showed no evidence of visual deficit postoperatively.

\section{Case 2}

This patient was a 76-year-old man who had an epi- 

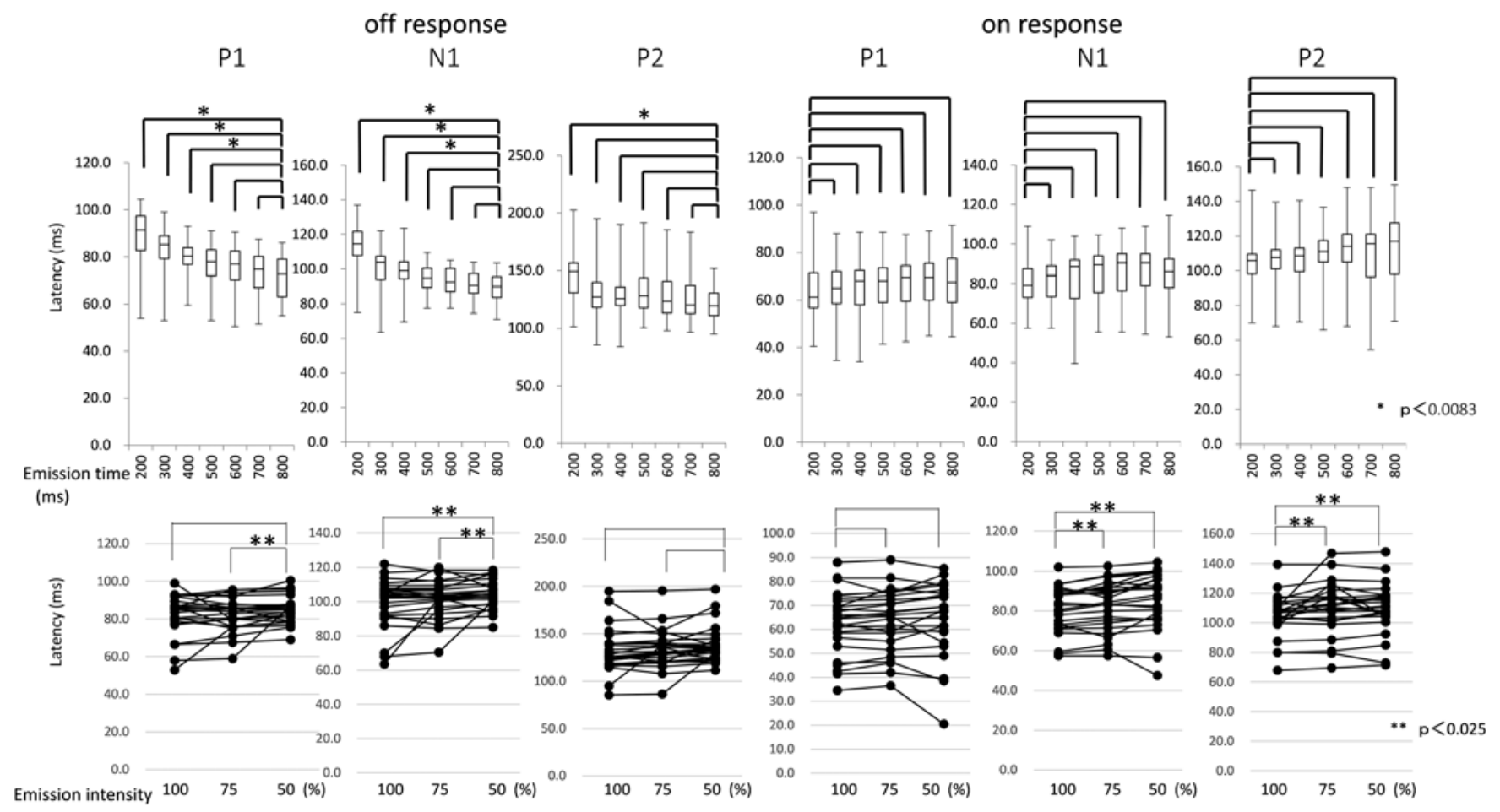

FIG. 5. The upper graphs show correlations between the peak latency during the light emission time and each peak latency. The left 3 graphs are data for the off response, and the right 3 graphs are data for the on response. The off response was analyzed by Mann-Whitney tests relative to $800 \mathrm{msec}$. The on response was analyzed by Mann-Whitney tests relative to $200 \mathrm{msec}$. The lower graphs show correlations between the peak latency and the emission quantity. The off response was analyzed by Wilcoxon signed-rank test relative to $50 \%$. The on response was analyzed by Wilcoxon signed-rank test relative to $100 \%$. ms $=m s e c$. ${ }^{*} p<$ 0.0083. ${ }^{* *} p<0.025$.

sode of transient cerebral ischemia. MR angiography revealed poor anterior circulation on the right side. Reduction of circulation reserve was observed in preoperative radioisotope studies. Angiography showed severe stenosis of the internal carotid artery and hypoplasia of $\mathrm{A}_{1}$. There was retrograde blood flow from the external carotid artery to the retina (Fig. 7, right). When carotid endarterectomy was performed, intermittent occlusion of the common carotid artery was carried out in order to establish the shunt tube. VEP monitoring with an emission quantity of 8000 Lx and an emission time of $500 \mathrm{msec}$ was continuously performed throughout the procedure; the amplitude was clearly decreased, and peak latency was delayed. In the results from the MO electrode, the amplitude of P1-N1 in the off response decreased from 1.35 to $0.99 \mu \mathrm{V}$ and that of N1-P2 decreased from 4.06 to $3.06 \mu \mathrm{V}$. The peak latencies for P1, N1, and P2 were also delayed from 76.5 to 84.5 $\mathrm{msec}$, from 88.5 to $94.5 \mathrm{msec}$, and from 108.0 to $114 \mathrm{msec}$, respectively (Fig. 7B). Normal amplitude was recovered after recanalization of blood flow following shunt setting. Peak latency was not delayed (Fig. 7C). This intermittent reduction was observed again when the common carotid artery was occluded during removal of the shunt tube. The patient did not experience any neurological deficits after surgery.

\section{Discussion}

This study focused on identification of valid factors retrieved from multiple uncertainties in VEPs using flash stimulation and application of these data during intraoperative monitoring. Therefore, it was necessary to separate the flash stimulus into the response to the start of light emission (on response) and the response to the cessation of light emission (off response).

In order to use a wave for VEP monitoring, the wave must be derived from the primary visual cortex. No prior studies have described such a method for intraoperative monitoring. In the present study, components that are considered to be from the primary visual cortex were estimated by statistical methods and were rather limited. Thus, although waves consisting of P1-N1-P2 in the on response and off response were considered to be derived from the primary visual cortex, both were inferred to be generated from different factors because they showed variations in the potential distribution.

It is also necessary to adjust the conditions for this wave to function as a monitoring tool. Reduction of individual differences is required in order for a parameter such as a wave to function as a monitoring tool. Two elements that can be adjusted for an emission stimulus are the light emission time and the quantity of light. This study showed that changing the emission time did not contribute to the reduction in individual differences in peak latency in the on response (Fig. 5). However, lengthening the light emission time reduced the individual differences in peak latency in the off response (Fig. 5). Based on these data, 

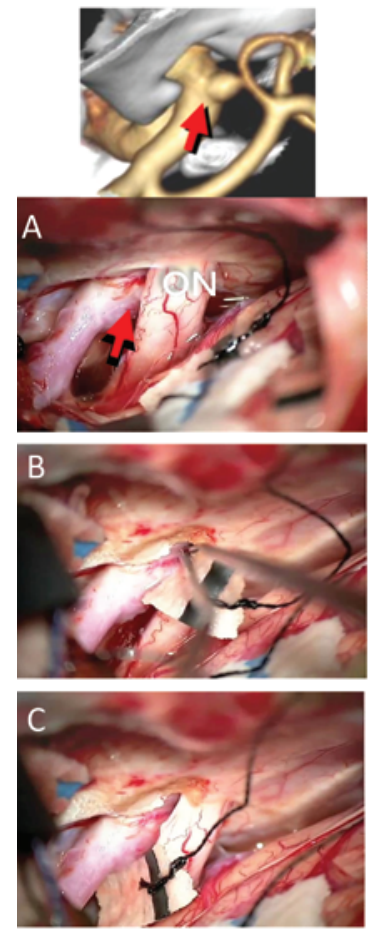

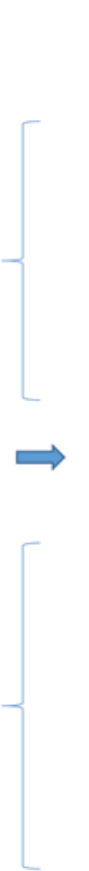

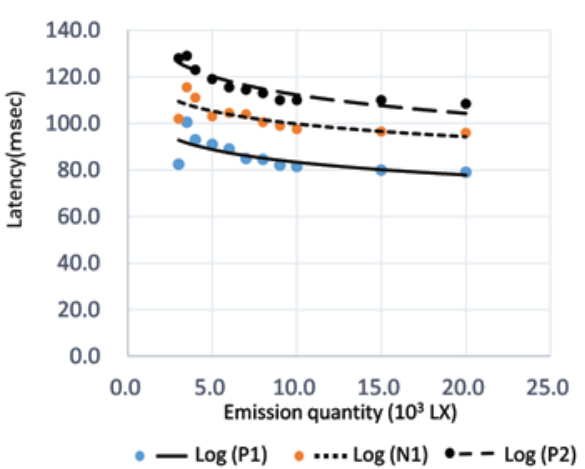

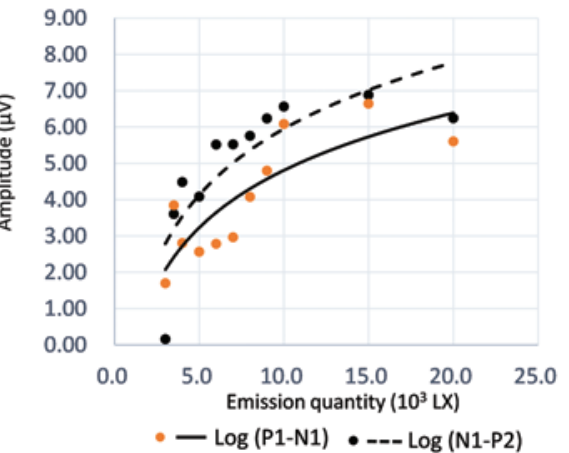

FIG. 6. Case 1. The left upper panel shows the surgical view in a 3D CT angiogram; the red arrow indicates the aneurysm. The intraoperative images show a surgical view of the optic nerve $(\mathrm{ON})$ and internal carotid artery $(\mathbf{A})$, with the red arrow indicating the aneurysm hidden by the optic nerve; confirmation of the proximal neck of the aneurysm by elevating the optic nerve (B); and the surgical field when the treatment was stopped $(\mathrm{C})$ based on the warning by the VEP monitor. The middle panel shows the VEP monitoring wave; the solid black arrow indicates a wave change after manipulation of the optic nerve. The graph in the right upper panel shows the change in peak latency with the artificial decrease in luminance quantity. The graph in the lower right panel shows changes in the amplitude with the artificial decrease in luminance quantity.

300-msec emission time was required to ensure separation of the on response and off response (Fig. 3). Moreover, an emission time of more than $500 \mathrm{msec}$ was required to reduce individual differences in the peak latency in the off response (Fig. 5).

Alternatively, the light emission intensity can be adjusted. In this study, changing the emission intensity caused opposite changes in the on response and off response. Specifically, the on response showed potential for reducing the individual differences in peak latency when the emission intensity was high, and the off response reduced the individual differences in peak latency when the emission intensity was lower. These results suggested that the flash stimulus did not exhibit stability, even after adjustment for the amount of light, because the flash stimulation included both the on and off responses simultaneously. Indeed, the on and off responses had conflicting stabilizing elements based on the quantity of light. Therefore, when a short flash was used, it was not possible to remove all instability.

In view of the above, stable monitoring could only be achieved when the off response was monitored using less light. In an actual surgical situation, allowing a small amount of light to continually reach the retina is much easier than allowing a large amount of light to continually reach the retina because of the interference of the thickness of the eyelid, the pupil diameter, the eye position, and the opacity of the cornea and vitreous humor. Moreover, maintaining a high light intensity may induce ocular damage due to heat. On the other hand, if there are many obstacles blocking light from reaching the retina, the off response will be more stable because individual differences are decreased as the quantity of light is reduced. Thus, the conditions present during surgery are not necessarily disadvantageous for the off response.

Finally, it is necessary to determine whether changes in visual function can be used as a monitoring tool. As shown in Case 1, the reaction decreased during surgical manipulation of the optic nerve. These changes could be reproduced by adjusting the amount of light, providing direct evidence that changes in the off response can be used as a monitoring tool. Moreover, the wave relationship of amplitude and latency during changes in emission intensity showed logarithmic changes rather than linear changes. Therefore, a decrease in the amplitude was observed as the input was reduced to a certain level or lower. Importantly, the wave changes introduced by manipulation of the optic nerve resolved when the manipulation was stopped. The reduction in amplitude caused by operative manipulation was comparable to a reduction in input by about one-third. Reduction of such a sensitive reaction will provide sufficient warning to the surgeon. Further studies are needed to determine whether this reduction is irreversible. Spe- 


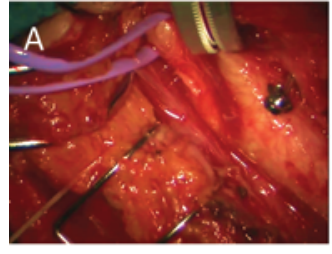

\section{Arterial occlusion}

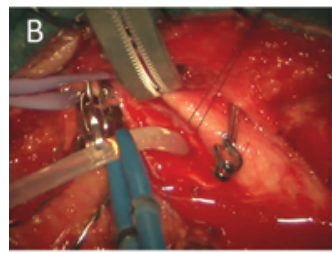

Recanalization

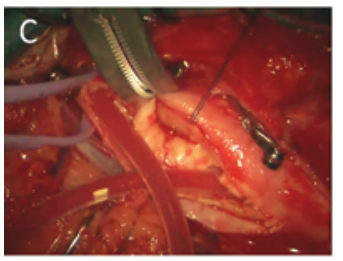

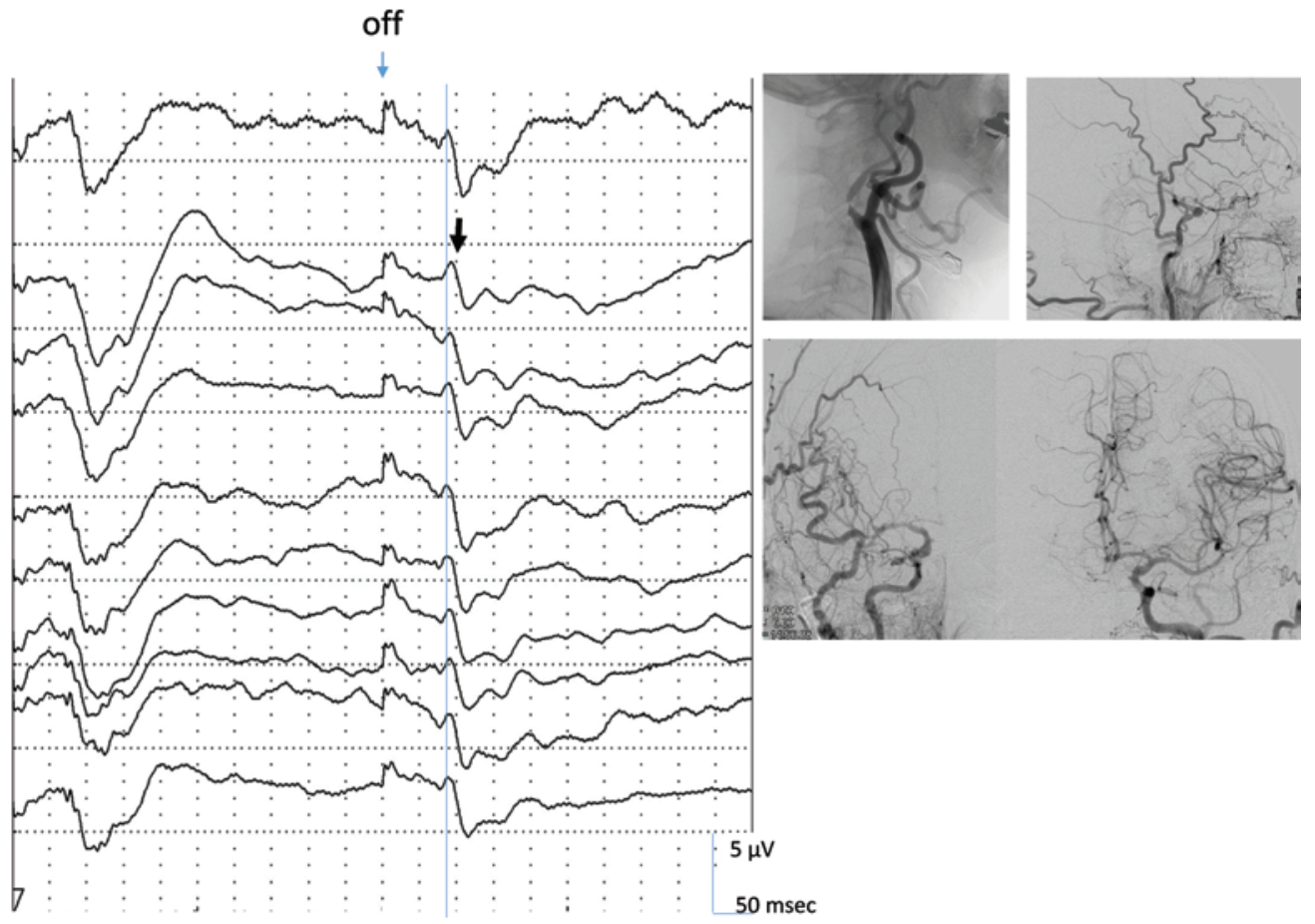

FIG. 7. Case 2. The intraoperative photographs show the exposure of the carotid bifurcation (A), insertion of the shunt tube after occlusion of the common carotid artery (B), and the re-establishment of blood flow into the internal carotid artery via the shunt tube (C). The middle panel shows the VEP monitoring wave from the MO electrode, and the black arrow indicates the wave change after arterial occlusion. The right panel shows preoperative angiographic images. The left upper image shows a cervical lesion. The upper right image shows the external carotid artery with retrograde perfusion to the retina. The lower image shows a cranial view of the right and left angiographies. The right $A_{1}$ shows hypoplasia.

cifically, a drop in the amplitude of the off response was apparent when the light quantity was less than $4000 \mathrm{Lx}$. Therefore, $8000 \mathrm{Lx}$ should be used as an optimal parameter for effective monitoring.

The reduction in amplitude observed in Case 2 was thought to reflect a decrease in blood flow to the retina. In this case, prolongation of the peak latency was also observed. However, because this was not a direct invasion, as in Case 1, these data showed that it was possible to monitor the effects of circulation affecting visual function. Thus, further studies are required to determine whether there are differences in how the wave changes between patients with blood circulation disorders of the retina and direct damage to the optic nerve.

\section{Conclusions}

This study demonstrated favorable stimulation during VEP monitoring using the off response, which did not require high levels of light stimulation. Moreover, it was possible to estimate the optimal stimulation level from this series of data. Specifically, a drop in the amplitude of the off response was apparent when the light quantity was less than $4000 \mathrm{Lx}$. These data suggest that $8000 \mathrm{Lx}$ should be used as an optimal parameter for effective monitoring. The method and interpretation of the results are simple and should be easily reproducible. Moreover, these results may explain why stable intraoperative VEP recording has not been possible until now. Suitable observation of the waveform generated during the off response is needed for intraoperative monitoring. Most importantly, for suitable monitoring, waves around $100 \mathrm{msec}$ were constructed in P1-N1-P2, and settings were adjusted to achieve a light emission quantity of $8000 \mathrm{Lx}$ and emission time of approximately $500 \mathrm{msec}$. This method may be applicable as a new method for VEP recording.

\section{References}

1. Chacko AG, Babu KS, Chandy MJ: Value of visual evoked potential monitoring during trans-sphenoidal pituitary surgery. Br J Neurosurg 10:275-278, 1996

2. Cedzich C, Schramm J: Monitoring of flash visual evoked potentials during neurosurgical operations. Int Anesthesiol Clin 28:165-169, 1990

3. Cedzich C, Schramm J, Fahlbusch R: Are flash-evoked visual potentials useful for intraoperative monitoring of visual pathway function? Neurosurgery 21:709-715, 1987

4. Cedzich C, Schramm J, Mengedoht CF, Fahlbusch R: Factors that limit the use of flash visual evoked potentials for surgical monitoring. Electroencephalogr Clin Neurophysiol 71:142-145, 1988

5. Chung SB, Park CW, Seo DW, Kong DS, Park SK: Intraoperative visual evoked potential has no association with postoperative visual outcomes in transsphenoidal surgery. Acta Neurochir (Wien) 154:1505-1510, 2012

6. Fisher RS, Raudzens P, Nunemacher M: Efficacy of intraop- 
erative neurophysiological monitoring. J Clin Neurophysiol 12:97-109, 1995

7. Freye E: Cerebral monitoring in the operating room and the intensive care unit - an introductory for the clinician and a guide for the novice wanting to open a window to the brain. Part II: Sensory-evoked potentials (SSEP, AEP, VEP). J Clin Monit Comput 19:77-168, 2005

8. Goto T, Tanaka Y, Kodama K, Kusano Y, Sakai K, Hongo K: Loss of visual evoked potential following temporary occlusion of the superior hypophyseal artery during aneurysm clip placement surgery. Case report. J Neurosurg 107:865-867, 2007

9. Harding GF, Bland JD, Smith VH: Visual evoked potential monitoring of optic nerve function during surgery. J Neurol Neurosurg Psychiatry 53:890-895, 1990

10. Kamio Y, Sakai N, Sameshima T, Takahashi G, Koizumi S, Sugiyama K, et al: Usefulness of intraoperative monitoring of visual evoked potentials in transsphenoidal surgery. Neurol Med Chir (Tokyo) 54:606-611, 2014

11. Kawaguchi T, Ogawa Y, Fujiwara S, Tominaga T: [A significant increase in intraoperative flash visual evoked potential amplitude during craniopharyngioma surgery-case report.] No Shinkei Geka 43:323-329, 2015 (Jpn)

12. Kodama K, Goto T, Sato A, Sakai K, Tanaka Y, Hongo K: Standard and limitation of intraoperative monitoring of the visual evoked potential. Acta Neurochir (Wien) 152:643648,2010

13. Kuroiwa Y, Celesia GG: Visual evoked potentials with hemifield pattern stimulation. Their use in the diagnosis of retrochiasmatic lesions. Arch Neurol 38:86-90, 1981

14. Lorenz M, Renella RR: [Intraoperative monitoring: visual evoked potentials in surgery of the sellar region.] Zentralbl Neurochir 50:12-15, 1989 (Ger)

15. Luo Y, Regli L, Bozinov O, Sarnthein J: Clinical utility and limitations of intraoperative monitoring of visual evoked potentials. PLoS One 10:e0120525, 2015 (Erratum in PLoS One 10:e0133819, 2015)

16. Nakagawa I, Hidaka S, Okada H, Kubo T, Okamura K, Kato $\mathrm{T}$ : [Effects of sevoflurane and propofol on evoked potentials during neurosurgical anesthesia.] Masui 55:692-698, 2006 (Jpn)
17. Nau HE, Hess W, Pohlen G, Marggraf G, Rimpel J: [Evoked potentials in intracranial operations: current status and our experiences.] Anaesthesist 36:116-125, 1987 (Ger)

18. Pastor J, Vega-Zelaya L, Pulido P, Garnés-Camarena O, Abreu A, Sola RG: Role of intraoperative neurophysiological monitoring during fluorescence-guided resection surgery. Acta Neurochir (Wien) 155:2201-2213, 2013

19. Raudzens PA: Intraoperative monitoring of evoked potentials. Ann N Y Acad Sci 388:308-326, 1982

20. Russ W, Krumholz W, Hempelmann G: [Visual evoked potentials (VEP) in anesthesia and intensive care.] Anaesthesist 33:154-160, 1984 (Ger)

21. Sasaki T, Itakura T, Suzuki K, Kasuya H, Munakata R, Muramatsu $\mathrm{H}$, et al: Intraoperative monitoring of visual evoked potential: introduction of a clinically useful method. J Neurosurg 112:273-284, 2010

22. Sasaki T, Nishijima M: [Preservation of visual function by intraoperative VEP monitoring: consideration from surgical procedures caused VEP changes.] No Shinkei Geka 41:961976, 2013 (Jpn)

23. Tobimatsu S, Celesia GG: Studies of human visual pathophysiology with visual evoked potentials. Clin Neurophysiol 117:1414-1433, 2006

24. Wiedemayer H, Fauser B, Armbruster W, Gasser T, Stolke D: Visual evoked potentials for intraoperative neurophysiologic monitoring using total intravenous anesthesia. J Neurosurg Anesthesiol 15:19-24, 2003

25. Wiedemayer H, Fauser B, Sandalcioglu IE, Armbruster W, Stolke D: Observations on intraoperative monitoring of visual pathways using steady-state visual evoked potentials. Eur J Anaesthesiol 21:429-433, 2004

\section{Disclosures}

The author reports no conflict of interest concerning the materials or methods used in this study or the findings specified in this paper.

\section{Correspondence}

Atsushi Sato, Koshirokubo 1313-1, Ina-shi, Nagano, Japan. email: asato@inahp.jp. 